\title{
Performance of Wireless Network using Network Coding over Store and Forward Technique
}

\author{
Mane Pallavi Ramesh \\ Department of Electronics and \\ Communication Engineering, \\ Manipal Institute of Technology, \\ Manipal University, Manipal
}

\author{
M. Sathish Kumar \\ Department of Electronics and \\ Communication Engineering, \\ Manipal Institute of Technology, \\ Manipal University, Manipal
}

\author{
Sudhakara G. \\ Department of Mathematics, \\ Manipal Institute of Technology, \\ Manipal University, Manipal
}

\begin{abstract}
In recent year's wireless networks has become a necessity rather than a luxury. Wireless networks suffer in providing high throughput for high end applications like multimedia etc. Network coding is proposed to improve the throughput of the network. In this paper, we apply network coding technique at the relay in wireless environment which is based on a simple XOR. It brings out the benefits of network coding over store and forward technique. The results highlight that using a simple XOR based network coding increases the normalized stable throughput compared to traditional store and forward routing.
\end{abstract}

\section{Keywords}

Network Coding, Wireless Networks, Stable Throughput, Packet Loss

\section{INTRODUCTION}

A wireless network plays a significant role to meet our basic need of providing connectivity with mobility. Current wireless networks struggle to meet throughput and reliability demands placed on them by today's wireless applications. Network Coding is proposed to be a solution to these problems. It introduces coding of packets either at source or at intermediate nodes to increase the throughput of the network. The technique is considered by several researchers as an extension of the traditional store and forward paradigm and can effectively reduce packet density in communication networks.

Current wireless networks are based on the principle of store and forward (SF) technique. Using SF technique the intermediate node simply buffers every packet and then forwards it individually without any modification. It is a known fact that wireless channels are noisy and also affected by fading which can result in packet losses.

\section{BACKGROUND}

R. Ahlswede et al. was the first one to propose the idea of network coding [1], a simple solution which has the potential to overcome limitations of SF strategy in the context of wireless networks. The basic idea of network coding is to mix information packets either at source nodes or at intermediate nodes thereby squeezing the information of several packets into one coded packet. This reduces the packet density in the network. Since the publication of these results in the year 2000 , network coding has attracted considerable attention from the communication networks research community [2-9].

Based on the level of implementation, network coding can be categorized as analog, symbol level, and packet level. Shengli Zhang et al. in [10], for the first time brought out the advantages of utilizing the physical layer signal level interference. The physical layer network coding was further investigated by Sachin Katti et al. in [11] and proposed a symbol level network coding, MIXIT. Packet level network coding, COPE is presented by Sachin Katti et al. in [12] and [13].

COPE was the first practical implementation of network coding architecture for networks with two user nodes with an intermediate access point (or relay), $\mathrm{X}$ topology, and cross topology. In the basic COPE model, routing was oblivious of coding opportunities. However, Sudipta Sengupta et. al., in [14] and [15] showed that throughput can be further increased over that of the basic COPE by making the routing of packets in a way that the router is aware of network coding opportunities. Along similar lines the work by Shravan Rayanchu et al. in [16] makes an effort to utilize the best coding opportunities with awareness of packet losses. The limitation of COPE is that it does not consider the packet priority information while computing coding opportunity was brought out by Kaikai Chi et al. in [17]. Consequently, they proposed modifications to COPE's queuing structure and proposed a new packet scheduling algorithm to assign priorities to the packets.

This paragraph contains a brief review of published literature on network coding which have used queuing models to evaluate various performance aspects of network coding. Yi Ma et al. in [18] presented a delay analysis and Omer $\mathrm{H}$ et al. in [19] explored the bandwidth and delay performance of network coding. Parimal Parag et al. gave comparison of network coding to classical routing for a butterfly network [20]. Yalin Evren Sagduyu et. al. in [21] discussed the throughput limitations of retransmission schemes and evaluated the stability benefits of network coding used for broadcasting over erasure channels. Daisuke Umehara et al., in [22] analyzed the throughput and packet delay of network coding in slotted ALOHA for a two hop wireless network with a single relay. Mohammad H. Amerimehr et al. in [23] analyzed the throughput of WLAN consisting of one relay node and six user nodes that implemented network coding only at relay. However their model considers error free transmission and hence in [24] packet loss due to link transmission errors are incorporated in the analysis. In this paper we present the throughput analysis with four user nodes and single relay.

\section{SYSTEM MODEL}

Consider a wireless network with 4 user nodes and one relay as shown in Fig. 1. The transmission range of any node is one hop. Every user node can listen to the packets that are sent to or from its neighboring node. Hence node 1 can listen to node 2 and node 4 . Similarly node 3 can listen to node 2 and node 3 , node 2 can listen to node 1 and node 3 , and node 4 can 
listen to node 1 and node 4 . Relay node is in the range of all user nodes. The packets to be delivered from node 1 to node 3 are to be forwarded by relay node. Similarly relay has to forward packets sent from node 3 to node 1 , node 2 to node 4 and node 4 to node 2 .

Using traditional store and forward technique relay the network uses four transmissions slots to exchange one packet from node 1 and node 3 . Using network coding relay can XOR the individual packets received from node 1 and node 3 and forward the coded (XORed) packet to both node 1 and node 3 . As node 1 knows its own packet, it can decode the coded packet upon its error free reception. Thus network coding here save one transmission time slot and bandwidth equivalent to one packet increasing the throughput of the network. Similar situation can arise when node 2 and node 4 has to exchange packets.

Each node is equivalently represented as a queue based on FIFO policy. Packet arrivals are assumed to be symmetric across all the user nodes in the network. The arrival rate at each user node is $\lambda$ and is uniformly distributed to all the other nodes in the network.

To proceed with the analysis, let $W$ and $W^{\prime}$ be the MAC contention window length at user and relay nodes respectively. A given user node which has a packet for transmission waits for a random number of time slots, which ranges from 0 to $(W-1)$, before it starts the transmission. Thus the service time $\mu$ is the average time taken to transmit a packet by a given user will be $(W+1) / 2$ times the time slot duration. Therefore, under the condition that a given user has a packet ready for transmission, the probability of transmitting that packet is $P_{t}=2 /(W+1)$. With the utilization factor expressed as $\rho$ for the user node, the probability of the user

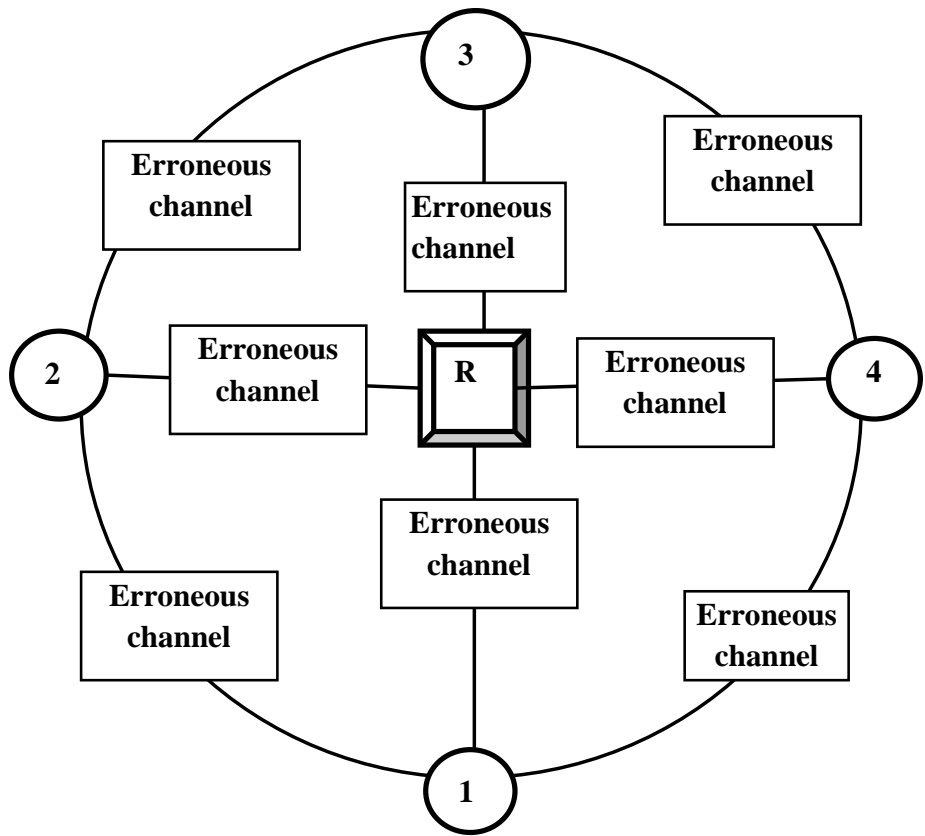

Fig. 1: 4 user nodes and 1 relay node wireless network

node transmitting packets is $\rho P_{t}$.

Similarly for the relay node, this probability will be $\rho^{\prime} P_{t}^{\prime}$ where, $\rho^{\prime}$ is the utilization factor of the relay node, service time $\mu^{\prime}=\frac{\left(W^{\prime}+1\right)}{2}$ and $P_{t}^{\prime}=\frac{2}{\left(W^{\prime}+1\right)}$.

\subsection{Analysis at user Node}

The mode of transmission is considered to be half duplex. MAC collisions may lead to packet losses. Every node has two neighboring user nodes and one relay in the transmission range. Hence a packet from node $k$, is successfully delivered to its neighboring user node (node $k-1$ or node $k+1$ ) if and only if the receiving node (node $k-1$ or node $k+1$ ) and its neighbors except node $k$ should not transmit any packet. To illustrate a packet to send from node 1 to node 1 is successfully delivered if node 2 , node 3 and relay node does not transmit any packets in the same slot as well as the packet sent from node 1 does not suffer from errors or erasures. Hence the probability of successful delivery of packet from a user node to its neighboring user node $P\left(D_{1}\right)$ is obtained as

$$
P\left(D_{1}\right)=\left(1-P_{t} \rho\right)^{2}\left(1-P_{t}^{\prime} \rho^{\prime}\right)(1-p)
$$

Similarly a packet sent from node 1 to node 3 , must be successfully received by relay node. In general a packet sent from node $\mathrm{k}$ to its peer node (node $k+2$ ) is successfully received by relay node if relay and all user nodes except node 1 should not transmit any packet in that transmission time slot and the packet does not suffer any errors or erasures. Hence the probability of successful delivery of packet from a user node to relay node $P(I)$ is obtained as

$$
P(I)=\left(1-P_{t} \rho\right)^{(n-1)}\left(1-P_{t}^{\prime} \rho^{\prime}\right)(1-p)
$$

Let $\alpha_{D_{1}}$ be the overall arrival rate of packets to be delivered from every user node to neighboring user node including the retransmission of lost packets. It can be shown that

$$
\alpha_{D_{1}}=\frac{\lambda}{4\left(1-P_{t} \rho\right)^{2}\left(1-P_{t}^{\prime} \rho^{\prime}\right)(1-p)}
$$

Similarly $\alpha_{D_{2}}$ be the overall arrival rate of packets to be delivered from every user node to its peer including the retransmission of lost packets. It can be shown that

$$
\alpha_{D 2}=\frac{\lambda}{4\left(1-P_{t} \rho\right)^{3}\left(1-P_{t}^{\prime} \rho^{\prime}\right)(1-p)}
$$

The overall arrival rate at user node $=2 \alpha_{D 1}+2 \alpha_{D 2}$. The utilization factor at every node is defined as the ratio of arrival rate to the service rate at that queue. Thus, the utilization factor of queue at a user node is

$$
\rho=\frac{\lambda}{4 \mu\left(1-P_{t} \rho\right)^{2}\left(1-P_{t}^{\prime} \rho^{\prime}\right)(1-p)}\left(1+\frac{2}{\left(1-P_{t} \rho\right)}\right)
$$

\subsection{Analysis at Relay Node}

Relay node is equivalently represented by a queue based on FIFO policy. This section presents the analysis at relay node with or without the implementation of network coding.

\subsubsection{Without Network Coding at Relay Node}

Relay node without the implementation of network coding has forward packets received from all user nodes individually to its appropriate destination user node. Again packets may be lost due to MAC collision or due to channel errors or erasures. A packet from relay node is delivered successfully to appropriate destination (user node) if and only if the destined user node and neighbors of destined user node are not 
transmitting any packet in the same time slot and the packet does not suffer any errors or erasures. Hence the probability of successful delivery of packets from relay node to user nodes is given by

$$
P(\text { relay })=\left(1-P_{t} \rho\right)^{3}(1-p)
$$

From every user node there is only one such packet requires the service of relay. The arrival rate of packets at relay is sum of packet arrivals from all user nodes that requires relay to forward their packets to appropriate user nodes including the retransmission from relay node. This arrival rate at relay node including the retransmission of lost packets is obtained as

$$
\alpha_{s f}=\frac{\lambda}{\left(1-P_{t} \rho\right)^{3}(1-p)}
$$

Hence utilization factor at relay node with simple store and forward technique is arrived at

$$
\rho_{s f}=\frac{\lambda}{\mu^{\prime}\left(1-P_{t} \rho\right)^{3}\left(1-p_{l}\right)}
$$

\subsubsection{With Network Coding at Relay Node}

With the implementation of network coding at relay, for every two received packets a coded packet is constructed by computing the XOR of these two packets. This effectively reduces the transmission of a packets at the by two. There is every chance that coded packet is not delivered or delivered with errors to both the destination nodes, which is to be retransmitted. If the coded packet is delivered successfully to only one of the two destination node, then it is subjected to retransmission using simple store and forward technique. With this model the ratio of overall arrival rate to service rate at relay node with network coding is given as

$$
\rho_{n c}=\frac{(n-3) \lambda\left[\left(3 P_{c 1}+4 P_{c 2}\right)-2\left(\left(1-P_{t} \rho\right)^{3}(1-p)\right]\right.}{2 \mu^{\prime}\left(1-P_{t} \rho\right)^{3}(1-p)\left[2-\left(1-P_{t} \rho\right)^{3}(1-p)\right]}
$$

We define stable throughput as the maximum arrival rate at the user nodes that the network can handle such that any further increase in the arrival rate makes the network unstable. That is, In other words throughput is measure of maximum arrival rate (packets per time slot) that the network can handle without dropping of packets at any of the network nodes. The throughput of the network when relay uses store and forward technique is obtained as

$$
\lambda_{s f}=\mu^{\prime} \rho_{s f}\left(1-P_{t} \rho\right)^{3}(1-p) ; \rho_{s f} \leq 1 \text { and } \rho \leq 1
$$

and throughput of the network when relay uses store and forward technique is obtained as with network coding

$$
\lambda_{n c}=\frac{\rho_{n c} 2 \mu^{\prime}\left(1-P_{t} \rho\right)^{3}(1-p)\left[2-\left(1-P_{t} \rho\right)^{3}(1-p)\right]}{\left[3-2\left(\left(1-P_{t} \rho\right)^{3}(1-p)\right]\right.}
$$

where $\rho_{n c} \leq 1$ and $\rho \leq 1$

\section{RESULTS AND DISCUSSION}

This section investigates the range of MAC contention window at user node and relay for which the system remains stable and thereby evaluates the maximum stable throughput of the network. The throughput variation is investigated for the packet $p$ up to $15 \%$.
Selecting appropriate values for contention window, the utilization factor at user node is solved for and used to compute the maximum throughput of the network as shown in figure 1 to figure 3 .

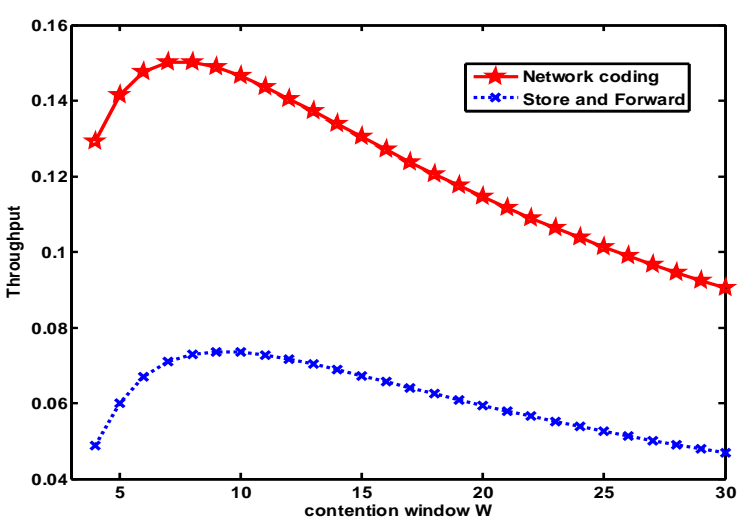

Fig 1: Normalized throughput with $p=0$

Figure 1 is the plot for normalized throughput when the transmission is error free and packet loss is only due to MAC collisions. It is seen that the throughput using network coding is much higher than simply using store and forward technique.

Figure 2 shows the maximum stable throughput as a function of MAC contention window with packet loss due to link transmission errors $p=0.1$. It is seen that network coding out performs store and forward technique.

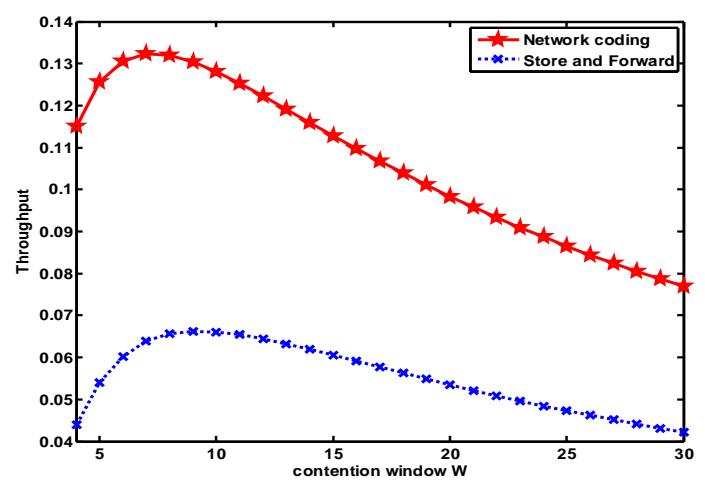

Fig 2: Normalized throughput with $p=0.1$

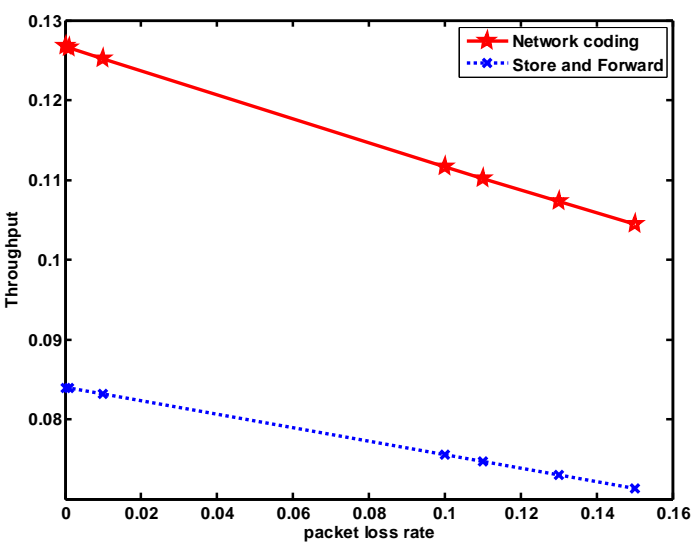

Fig 3: Normalized throughput with fixed the contention window $W$ to 10 . 
Finally figure 3 shows the throughput as a function of packet loss rates due to link transmission errors. The benefits of network coding are seen clearly from the plots. It is measured as percentage throughput gain defined by equation (2.12) and is as shown in figure 4. Aproximately $45 \%$ to $55 \%$ and indicated as shown in figure 4.

$$
\left(\lambda_{n c}-\lambda_{s f}\right) / \lambda_{s f} * 100
$$

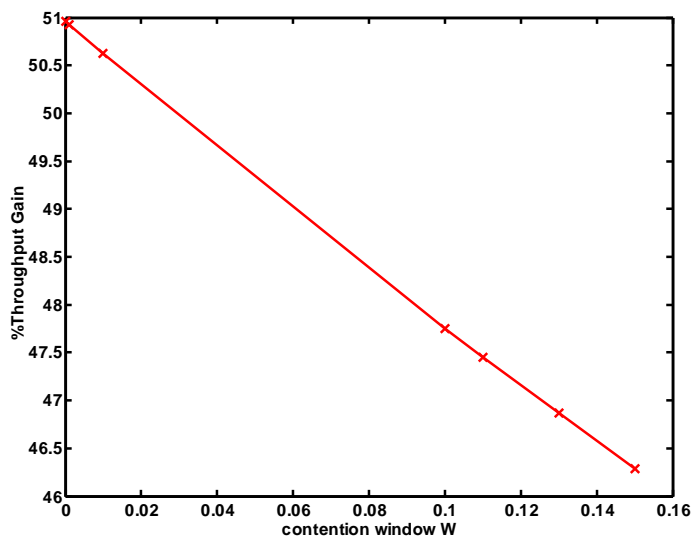

Fig 4: Percent throughput gain of network coding over store and forward technique setting the $\mathrm{MAC}$ window to 10 , and for the range of .packet loss due to link transmission errors ranging 0 to $15 \%$

\section{CONCLUSION}

This paper brings out the benefits of network coding in a wireless network consisting of four user nodes and one relay. Network coding is based on a simple XOR to combine packet whenever packets are to be exchanged between the user nodes. Network coding outperforms the traditional store and forward technique. It is worth investigating benefits of network coding with varying number of user nodes. Our future work is to apply the model to investigate the performance of wireless network that consist multiple user nodes and bring out the benefits of network coding over store and forward.

\section{REFERENCES}

[1] Rudolf Ahlswede, Ning Cai, Shuo-Yen Robert Li, and Raymond W. Yeung, "Network information flow," IEEE Trans. Inform. Theory, Vol.46, No. 4, pp. 1204-1216, July 2000.

[2] Rudolf Ahlswede, Ning Cai, Shuo-Yen Robert Li, and Raymond W. Yeung, "Network information flow," IEEE Trans. Inform. Theory, Vol.46, No. 4, pp. 1204-1216, July 2000.

[3] Shuo-Yen Robert Li, Raymond W. Yeung, and Ning Cai, "Linear Network Coding," IEEE Trans. Information Theory, Vol. 49, No. 2, pp. 371-381, February 2003.

[4] Randall Dougherty, Christopher Freiling, and Kenneth Zeger, "Linearity and Solvability in Multicast Networks", IEEE Trans. Information Theory, Vol. 50, No. 10, pp 2243-2256 October 2004.

[5] Randall Dougherty, Christopher Freiling, and Kenneth Zeger, "Insufficiency of Linear Coding in Network Information Flow", IEEE Trans. Information Theory, Vol. 51, No. 8, pp. 2745-2759, August 2005.
[6] Christina Fragouli, JeanYves Le Boudec, and Jorg Widmer, "Network Coding: An Instant Primer", Vol 36, No. 1, ACM SIGCOMM on Computer Communication, pp. 63-68, January 2006

[7] Christina Fragouli, Dina Katabi, Athina Markopoulou, Muriel Medard, Hariharan Rahul., "Wireless Network Coding: Opportunities \& Challenges," . IEEE Military Communications Conference, MILCOM'07, pp.1-8, October 2007

[8] Ralf Koetter and Muriel Medard, "An Algebraic approach to network coding", IEEE/ACM Trans. on Networking, Vol. 11, No. 5, pp. 782-795, October 2003.

[9] Philip A. Chou, Yunnan Wu, and Kamal Jain, "Practical Network Coding," in Proc. 41st Allerton Conf. on Comm. Control and Computing, Monticell, IL, USA, Vol. 1, pp. 1-10, October 2003.

[10] Shengli Zhang, Soung-Chang Liew, Patrick P.Lam, "Physical-layer network coding", in Proc. of ACM MOBICOM '06, pp. 358-365, September 2006.

[11] Sachin Katti, Shyamnath Gollakota and Dina Katabi, "Embracing Wireless Interference: Analog Network Coding”, SIGCOMM'07, pp. 397-408, August 2007, Kyoto, Japan

[12] Sachin Katti, Dina Katabi, Hari Balakrishnan, and Muriel Medard, "Symbol-level network coding for wireless mesh networks", ACM SIGCOMM'08, Conf. on Data communication, Vol. 38, No. 4, pp. 401-412, August 2008.

[13] Sachin Katti, Hariharan Rahul, Wenjun Hu, Dina Katabi, Muriel Medard, Jon Crowcroft, "XOR $s$ in the air: practical wireless network coding", IEEE/ACM Trans. on Networking, Vol. 16, No.3, pp497-510, June 2008.

[14] Sudipta Sengupta, Shravan Rayanchu, Suman Banerjee, "An Analysis of Wireless Network Coding for Unicast Sessions: The Case for Coding-Aware Routing", IEEE Int. Conf. on Computer Communications, pp. 1028-1036, INFOCOM, May 2007.

[15] Sudipta Sengupta, Shravan Rayanchu, Suman Banerjee, "Network Coding - Aware Routing in wireless Networks", IEEE/ACM Tran. On Networking, Vol.18, No.4, pp. 1158-1170, August 2010.

[16] Shravan Rayanchu, Sayandeep Sen, Jianming Wu, Suman Banerjee and Sudipta Sengupta, "Loss-Aware Network Coding for Unicast Wireless Sessions: Design, Implementation, and Performance Evaluation", ACM SIGMETRICS pp. 85-86, June 2008.

[17] Kaikai CHI, Xiaohong JIANG, and Susumu HORIGUCHI, "A More efficient COPE architecture for Network coding in Multihop wireless Networks", IEICE Trans. Commun. Vol. E92-B, No.3, pp. 766-774, March 2009

[18] Yi Ma; Wei Li; Pingyi Fan; Xuming Liu, "Queuing model and delay analysis on network coding," Communications and Information Technology, 2005. ISCIT 2005. IEEE International Symposium on , Vol.1, pp.112,115, 12-14 Oct. 2005

[19] Omer H. Abdelrahman, Erol Gelenbe, "Queuing Performance under Network Coding", ITW 2009, Greece, pp. 135-139, June 2009. 
[20] Parimal Parag and Jean-Francois Chamberland, "Queueing Analysis of a Butterfly Network for Comparing Network Coding to Classical Routing", IEEE Trans. On Information Theory, Vol.56, No.4, pp. 18901908 , April 2010.

[21] Yalin Evren Sagduyu and Antony Ephremides, “On Broadcast Stability of Queue-Based Dynamic Network Coding Over Erasure Channels", IEEE Tran. On Information Theory, Vol. 55, No. 12, pp. 5463-5478, Dec 2009.

[22] Daisuke Umehara, Tomoya Hirano, Satoshi Denno, Masahiro Morikura, and Takatoshi Sugiyama, "Wireless
Network Coding in Slotted ALOHA with Two-Hop Unbalanced traffic", IEEE on Selected Areas in Communications, Vol. 27, No. 5, pp. 647-661, June 2009

[23] Mohammad H. Amerimehr, Farid Ashtiani and Mohammad B. Iraji, "An Analytical Approach for Throughput Evaluation of Wireless Network Coding", IEEE ICC pp. 1-5, 2009.

[24] Pallavi R. Mane, M. Sathish Kumar, Sudhakar G., and Ghanashyama Prabhu, "Throughput Analysis of WLAN with Network Coding", IJFCC, International Journal of Future Computer and Communication, Vol. 2, No. 1 pp. 23-26, February 2013 